\title{
CircNT5E-a promising new player in glioblastoma tumorigenesis
}

\author{
Tomasz Powrózek, Teresa Małecka-Massalska \\ Department of Human Physiology, Medical University of Lublin, Lublin, Poland \\ Correspondence to: Tomasz Powrózek, PhD. Department of Human Physiology, Medical University of Lublin, Radziwiłłowska 11, 20-080 Lublin, \\ Poland. Email: tomaszpowrozek@gmail.com. \\ Comment on: Wang R, Zhang S, Chen X, et al. CircNT5E Acts as a Sponge of miR-422a to Promote Glioblastoma Tumorigenesis. Cancer Res \\ 2018;78:4812-25.
}

Submitted Sep 16, 2018. Accepted for publication Oct 09, 2018.

doi: $10.21037 /$ tcr.2018.10.09

View this article at: http://dx.doi.org/10.21037/tcr.2018.10.09

Glioblastoma (GBM) is the most common primary malignant brain neoplasm, and is characterized by high aggressiveness and unfavorable prognosis. Generally, even with surgical intervention followed by irradiation, the median overall survival for GBM usually does not exceed 15 months. The disease prognosis depends especially on the tumor stage, size and location in relation to the anatomic structures of the central nervous system. The few treatment options that can be applied, such as the afore mentioned surgery with concurrent temozolomide-assisted radiation, along with other, supposedly more promising personalized therapies, still have unsatisfactory outcomes or demonstrate unacceptable side effects. Regarding the molecular profile of GBM, this type of tumor demonstrates a complex genetic pattern including alteration both in gene expression and numerous signaling pathways. Among studied cases, the most prevalent molecular alterations were found as follows: overexpression of epidermal growth factor receptor (EGFR), phosphate and tensin homologue gene (PTEN) mutations, methylation of O-6-Methylguanine-DNA Methyltransferase gene (MGMT), loss of chromosome 10q, and over-activation of tumor protein p53 (TP53) signaling. Perhaps in the near future, better understanding of the molecular profile of GMB will significantly improve disease prognosis and treatment outcomes, but as of today, many potential molecular agents have been unsuccessful in conducted clinical trials (1-5).

The genetic background and oncogenic trigger mechanisms which could be involved in the promotion of GBM tumorigenesis remain unclear. However, the most recent achievements in molecular biology seem to shed new light on the initiation of various neoplasm developments, including GBM. According to the latest research, tumorigenesis can be promoted and maintained by a group of non-coding RNA (ncRNA) molecules, especially microRNA (miRNA), long non- coding RNA (lncRNA) and the recently identified circular RNA (circRNA) $(6,7)$. circRNAs possess significant capabilities in gene regulation, and therefore have been carefully investigated as potential initiators in the development of various human diseases. The leading function of circRNA is their activity as miRNA sponges; hence, circRNA expression might be related to the inhibition of miRNA regulatory pathways $(8,9)$. Several circRNA have very lately been identified as promising "missing pieces" in the development of silicosis (circZC3H4 binds miRNA-212), hepatocellular carcinoma (circMTO1 binds miRNA-9), colorectal cancer (circCCDC66 binds miRNA-33b, 93 and 185) and others (10-12).

Interestingly, the mutual relationship among ncRNA has been discovered, which could provide an encouraging new approach to oncogenesis. This hypothesis was developed by Wang et al. who carefully explored the ncRNA profile in GBM (13). These researchers studied the role of miRNA-422a in GBM in detail (14). The pro-oncogenic function of this molecule was also proven in other studies of head and neck cancer, lung cancer and glioma (15-17). Wang et al. believe that circRNA and lncRNA act as efficient miRNA sponges that inhibit their expression. To confirm these assumptions, they used cell cultures, animal model, and glioma samples collected from patients, and processed these with various methods including microarray technique, immunochemistry, FISH and CRISPR/Cas9 technology. The experiments revealed over 350 of the circRNA and IncRNA overexpressed in miRNA422a downregulated samples. Then, using bioinformatics tools, the 38 circRNA/lncRNA with the highest potential of miRNA-422a sponging were selected and screened. Following study protocol, novel circRNA, the circNT5E was identified as a potential novel oncogene in GBM etiopathogenesis. The subsequent experiments confirmed its oncogenic potential. Notably, using the FISH technique, the circNT5E was 


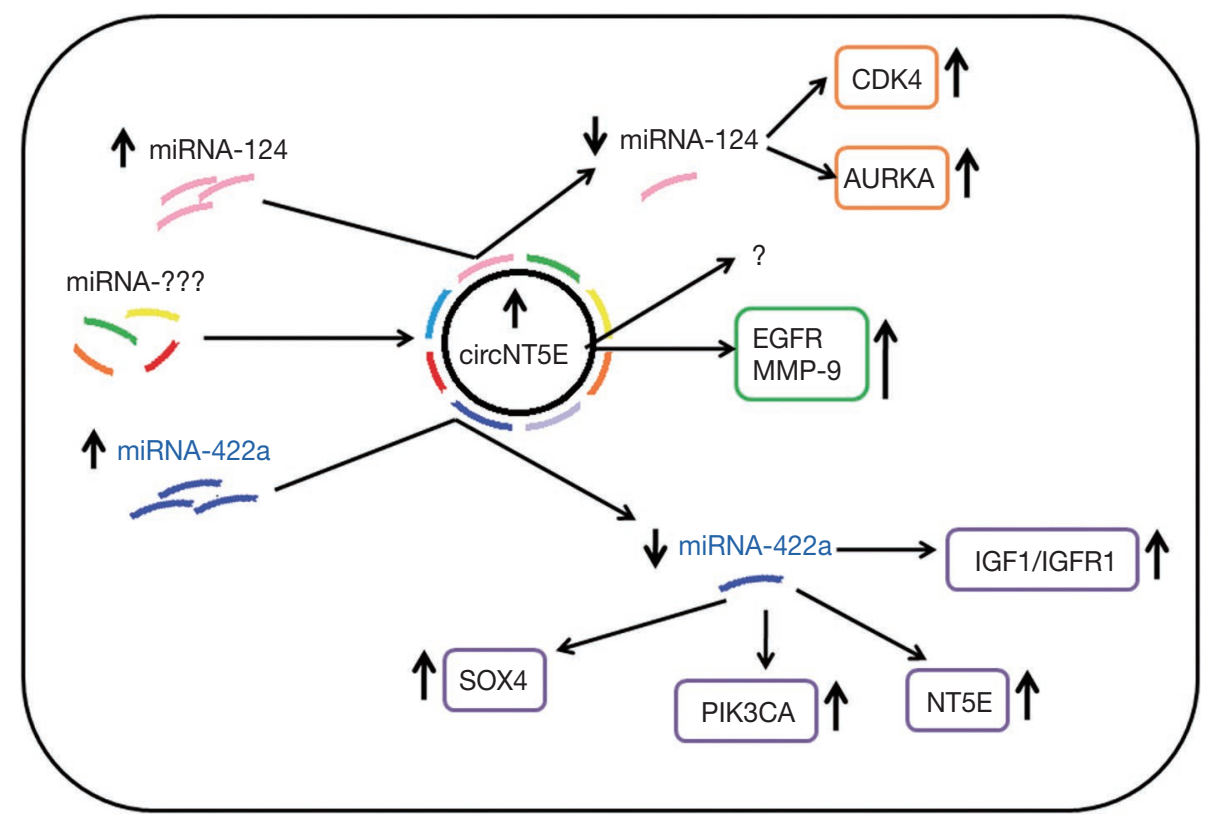

Figure 1 Putative role of circNT5E in GBM tumorigenesis. GBM, glioblastoma.

found in each set of studied samples and normal brain tissues; however, its expression positively correlated with a disease stage. Moreover, circRNA level was significantly higher than in healthy tissues. Results obtained in two cell lines (U87 and U251) showed that mimic of circNT5E promoted cell proliferation, migration and viability, whereas knock-down of studied circRNA demonstrated reverse effects. These findings emphasized its role as in vitro promoter of GMB, and therefore these results also required confirmation in vivo. The mice model confirmed this previous observation. Mice treated with circNT5E (mimic) demonstrated increased growth, size and mass of the tumor. The IHC revealed that circRNA promoted activation of the EGFR pathway and metalloprotease-9 (MMP-9) expression in the lesions. Conversely, mice treated with si-circNT5E (inhibitor) demonstrated inhibition of tumor development and reduced EGFR and MMP-9 expression. Wang et al. also proved that circNT5E acts as a sponge for miRNA-422a by reducing its expression. Studied circRNA affected the GBM cells proliferation, migration and invasiveness partly by miRNA-422a sponging, what was observed in cell lines. Two more interesting observations were noted. First, the specific brain-enriched RNA-editing enzyme ADARB2 was involved in the biogenesis of circNT5E in GBM. Second, the circNT5E/miRNA-422a axis upregulated NTSE, SOX4, PI3KCA, p-Akt, and p-Smad2 levels in GBM cells, whereas circNT5E knockdown downregulated NTSE, SOX4, PI3KCA, p-Akt, and p-Smad2 levels. The study demonstrated the significant role of circNT5E in GBM tumorigenesis both in vitro and in vivo, although these results require further investigation in a larger set of patients' samples. Postulated mechanism of circNT5E action is presented in Figure 1.

To conclude, the discussed study revealed a novel potential trigger mechanism involved in GBM tumorigenesis. A better understanding of its activity could be considered an interesting molecular target for future molecular agents. Moreover, the mutual relationship among different groups of ncRNA was demonstrated: in the case of miRNA-422a the particular $\operatorname{lncRNA} /$ circRNA were upregulated. It is also worth noting the limitations of discussed study. Due to molecular complexity of GBM, it is rather unlikely that knockdown of the single miRNA is a major trigger mechanism of tumorigenesis. How the authors also provided, the one circRNA can sponge variety of miRNA, and even is able to sponge a few molecules at the same time. They found 20 binding sites for miRNAs related to circNT5E, and currently we know effect of only miRNA-422a. This phenomenon suggests that probably on an early step of GBM tumorigenesis large number of miRNAs are inhibited by matching circRNA. Therefore, the close relationship between circRNA and miRNA should be further investigated especially in patients' samples. Eventually, all selected couples of circRNA/miRNAs need to be assessed together in one large study to establish their hierarchy in GBM tumorigenesis. The study also highlights the fact that there is still a lot to discover, 
as only one (circNT5E/miRNA-422a), out of possibly many more connections was identified. Perhaps other couples might prove to be more specific to GBM, but it has not been explored yet. In addition, the increase of IncRNA level, which was parallel to the increase of circRNA expression, demonstrates the complexity of GBM tumorigenesis. The question is if this mechanism is only related to GBM tumorigenesis or if it also possibly contributes to the promotion of other neoplasms. The mechanism for circRNA action is still in dispute and not fully understood, and therefore needs further strenuous investigation. New players have entered the cancer field, and we should remain critical and carefully observe their progress.

\section{Acknowledgments}

Funding: None.

\section{Footnote}

Provenance and Peer Review: This article was commissioned and reviewed by the Section Editor Chunlin Ou (Cancer Research Institute of Central South University, Changsha, China).

Conflicts of Interest: Both authors have completed the ICMJE uniform disclosure form (available at http://dx.doi. org/10.21037/tcr.2018.10.09). The authors have no conflicts of interest to declare.

Ethical Statement: The authors are accountable for all aspects of the work in ensuring that questions related to the accuracy or integrity of any part of the work are appropriately investigated and resolved.

Open Access Statement: This is an Open Access article distributed in accordance with the Creative Commons Attribution-NonCommercial-NoDerivs 4.0 International License (CC BY-NC-ND 4.0), which permits the noncommercial replication and distribution of the article with the strict proviso that no changes or edits are made and the original work is properly cited (including links to both the formal publication through the relevant DOI and the license). See: https://creativecommons.org/licenses/by-nc-nd/4.0/.

\section{References}

1. Davis ME. Glioblastoma: Overview of Disease and Treatment. Clin J Oncol Nurs 2016;20:S2-8.

2. Weller M, van den Bent M, Hopkins K, et al. EANO guideline for the diagnosis and treatment of anaplastic gliomas and glioblastoma. Lancet Oncol 2014;15:e395-403.
3. Alifieris C, Trafalis DT. Glioblastoma multiforme:

Pathogenesis and treatment. Pharmacol Ther. 2015;152:63-82.

4. Verhaak RG, Hoadley KA, Purdom E, et al. Integrated genomic analysis identifies clinically relevant subtypes of glioblastoma characterized by abnormalities in PDGFRA, IDH1, EGFR, and NF1. Cancer Cell 2010;17:98-110.

5. Wilson TA, Karajannis MA, Harter DH. Glioblastoma multiforme: State of the art and future therapeutics. Surg Neurol Int 2014;5:64.

6. Anastasiadou E, Jacob LS, Slack FJ. Non-coding RNA networks in cancer. Nat Rev Cancer 2018;18:5-18.

7. Batista PJ, Chang HY. Long noncoding RNAs: cellular address codes in development and disease. Cell 2013;152:1298-307.

8. Greene J, Baird AM, Brady L, et al. Circular RNAs: Biogenesis, Function and Role in Human Diseases. Front Mol Biosci 2017;4:38.

9. Barrett SP, Salzman J. Circular RNAs: analysis, expression and potential functions. Development 2016;143:1838-47.

10. Yang $X$, Wang J, Zhou Z, et al. Silica-induced initiation of circular ZC3H4 RNA/ZC3H4 pathway promotes the pulmonary macrophage activation. FASEB J 2018;32:3264-77.

11. Han D, Li J, Wang H, et al. Circular RNA circMTO1 acts as the sponge of microRNA-9 to suppress hepatocellular carcinoma progression. Hepatology 2017;66:1151-64.

12. Hsiao KY, Lin YC, Gupta SK, et al. Noncoding Effects of Circular RNA CCDC66 Promote Colon Cancer Growth and Metastasis. Cancer Res 2017;77:2339-50.

13. Wang R, Zhang S, Chen X, et al. CircNT5E Acts as a Sponge of miR-422a to Promote Glioblastoma Tumorigenesis. Cancer Res 2018;78:4812-25.

14. Liang H, Wang R, Jin Y, et al. MiR-422a acts as a tumor suppressor in glioblastoma by targeting PIK3CA. Am J Cancer Res 2016;6:1695-707.

15. Bonnin N, Armandy E, Carras J, et al. MiR-422a promotes loco-regional recurrence by targeting NT5E/CD73 in head and neck squamous cell carcinoma. Oncotarget 2016;7:44023-38.

16. Molina-Pinelo S, Gutiérrez G, Pastor MD, et al. MicroRNA-dependent regulation of transcription in nonsmall cell lung cancer. PLoS One 2014;9:e90524.

17. Wang H, Tang C, Na M, et al. miR-422a Inhibits Glioma Proliferation and Invasion by Targeting IGF1 and IGF1R. Oncol Res 2017;25:187-94.

Cite this article as: Powrózek T, Małecka-Massalska T. CircNT5E-a promising new player in glioblastoma tumorigenesis. Transl Cancer Res 2018;7(6):1341-1343. doi: 10.21037/tcr.2018.10.09 